\title{
The Influential Factors on Capital Structure: A Study on Portuguese High Technology and Medium-High Technology Small and Medium-Sized Enterprises
}

\author{
Yehui Tong ${ }^{1} \&$ Zélia Serrasqueiro ${ }^{2}$ \\ ${ }^{1}$ School of Accounting, Nanjing University of Finance and Economics, Nanjing, China \\ ${ }^{2}$ Department of Management and Economics, Faculty of Human and Social Sciences, University of Beira Interior, \\ Covilhã, Portugal \\ Correspondence: Yehui Tong, School of Accounting, Nanjing University of Finance and Economics, NO.3 Wenyuan \\ Road, Xianlin College Town, Nanjing 210023, Jiangsu, China. Tel: 86-180-9420-3897.
}

Received: January 21, 2020

Accepted: February 24, 2020

Online Published: June 28, 2020

doi:10.5430/ijfr.v11n4p23

URL: https://doi.org/10.5430/ijfr.v11n4p23

\begin{abstract}
Using the panel data model, this paper studies the influential factors on the capital structure of small and medium-sized enterprises (SMEs) in high and medium-high technology manufacturing sectors in Portugal. In particular, the total sample is further classified into young SME group and mature SME group for observing the similarities and differences. The research results show that firm size, profitability, firm age, and industry sector impact much on the capital structure and debt ratios; on the other hand, the impacts of tangible assets, intangible assets, and growth are not as strong as the previous factors. The differences of the impacts on young and mature SMEs are mainly shown by growth, intangible assets and industry sector. In particular, intangible assets show more statistical significance in young SMEs compared to mature SMEs, and intangible assets tend to be positively related to long-term debt especially in young SME group; this may reflect the positive attitude of financial institutions on the value of intangibles in generating future benefits for high and medium-high technology young firms. Besides, the findings tend to support the pecking order theory more than the trade-off theory regarding the high and medium-high technology manufacturing SMEs here.
\end{abstract}

Keywords: capital structure, small and medium-sized enterprises, high technology and medium-high technology

\section{Introduction}

Financing process (including the form and sources of financing studied as capital structure) is of significance for company management, because this is closely related to financial continuity and then the competitiveness of firms (Šarlija \& Harc, 2012). In particular, capital structure can reflect the information of the type of capital, the liquidity of funds, and business stability (Horváthová \& Mokrišová, 2017). About sixty years ago, the seminal research on capital structure was done by Modigliani and Miller (1958), as stated by Van Caneghem and Van Campenhout (2012); notwithstanding a number of studies in literature since then, the determinants of corporate capital structure is still argued by the researchers in financial economics (Rauh \& Sufi, 2010). Using panel data models, this paper studies the influential factors on the capital structure of small and medium-sized enterprises (SMEs) in high and medium-high technology manufacturing sectors in Portugal.

SMEs now play an important role in economic development. For instance, as pointed out by Andreeva et al. (2016), 99 percent of the total enterprises were SMEs that contributed to more than two thirds of the workforce and 58 percent of the total value added in European Union in 2011; in Portugal, as pointed out by Pais and Gama (2015), SMEs contributed to 77 percent of the jobs and more than 50 percent of the total turnover in 2011. However, small firms are more likely to suffer financial constrains (Jõeveer, 2013). What is more, compared to small firms in general, high technology small firms may face more financing problems because of more risks (such as the uncertainty of the market for the product or service) and then show higher default rates (Westhead \& Storey, 1997); in fact, it is the research and development $(\mathrm{R} \& \mathrm{D})$ projects of this kind of SMEs that generate many problems of information asymmetry between entrepreneurs and external investors, which further cause serious financial constraints (Pederzoli, Thoma, \& Torricelli, 2013). Therefore, it is necessary to study the capital structure of SMEs in high and 
medium-high technology sectors.

The research of corporate capital structure is regarding the relationship between the debt and equity sources and is reflected by the debt to equity ratio in the financing choice (Serghiescu \& Văidean, 2014; Strýčková, 2015). Furthermore, Bubic and Susak (2016) state that, aside from ownership (internal and external sources), financing sources can also be classified according to the maturity. In fact, exploring the impacts of debt maturity on firm's financial structure is also an important research line especially for small firms, since it is more difficult for small firms to obtain long-term debt as external financing (Pindado, Rodrigues, \& de la Torre, 2006). Hence this paper also examines long-term debt and short-term debt in order to find the differences in the influential factors. Thus, the contributions of this paper not only include finding important influential factors on capital structure to high and medium-high technology SMEs (which would be helpful to SME managers), but also contain studying the impacting factors on debt maturity structure. The following parts of this paper are composed of: literature review, data, variables, and methodology, results and discussion, and conclusions.

\section{Literature Review}

Capital structure has been researched for more than half a century; however, as stated by Myers (2001), there should be no expectation of universality in the theory of debt-equity choice. Researchers have proposed a series of theories regarding the optimal capital structure (Mihaela \& Claudia, 2017). The first one is the theory of Modigliani and Miller (1958) showing the irrelevance between capital structure choices and firm's investment decisions in perfect markets without taxes and transaction costs (Balakrishnan \& Fox, 1993). However, capital markets are imperfect in the real world, due to the problems of (for example) adverse selection, moral hazard, agency conflicts, and taxes (Dasilas \& Papasyriopoulos, 2015); thus, Modigliani and Miller (1963) took tax deductibility for interest expenses into consideration and proposed that increasing debt can increase the value of a firm (Vatavu, 2015). After the theories of Modigliani and Miller (1958; 1963), as stated by Rauh and Sufi (2010), there are two leading theories in capital structure research: the trade-off theory and the pecking-order theory.

\subsection{The Trade-off Theory and the Pecking Order Theory}

As pointed out by Bukalska (2019), the trade-off theory is developed on the basis of a series of research work-including the research of Modigliani and Miller (1963) about debt-tax shield, the research of Warner (1977) about bankruptcy and financial distress costs, and the research of Jensen and Meckling (1976) about agency costs; and, according to the trade-off theory, there exists an optimal capital structure that would be achieved by balancing the relation between the debt advantages (tax advantages) and costs (financial distress and agency costs). However, debt-tax shield is less used by SMEs because of lower profitability and higher risk of financial distress (Mac an Bhaird \& Lucey, 2010). As for the firms with large amount of intangible assets (such as high-tech firms), there would be higher costs of financial distress compared to the firms with large amount of tangible assets (Psillaki \& Daskalakis, 2009). Therefore, the relationship between the tangibility of assets and leverage should be positive, and the assumption of this positive relationship is supported by both the trade-off theory and the pecking order theory because of the function of tangible assets working as collateral to reduce potential bankruptcy costs as well as the effect in mitigating the problems of asymmetry information to creditors (Cwynar, Cwynar, \& Dankiewicz, 2015).

Based on information asymmetries between the company and the market, Myers (1984) and Myers and Majluf (1984) proposed the pecking-order theory which says that there is an order (firstly internally generated funds; secondly debt; lastly equity) for firm's financing (Acedo-Ramírez, Ayala-Calvo, \& Rodríguez-Osés, 2013); so it does not support the existence of an optimal capital structure (Ramalho \& da Silva, 2009). The pecking order theory is especially relevant to SMEs, as information opaque and adverse selection problems commonly exist in SMEs (Daskalakis \& Psillaki, 2008). As for the high-tech or innovative firms, Aghion et al. (2004) state that, because of more problems of asymmetric information between insiders and outsiders for the more innovative firms, the costs of issuing outside equity would be higher; so, according to the pecking order theory, the more innovative firms tend to be more reliant on external financing.

\subsection{Some Other Related Factors (Firm Size, Age, Profitability, Growth and Industry Sector)}

Firm size is an important factor impacting on debt. Because of the diversification of large companies and the negative relationship between size and bankruptcies, the trade-off theory identifies a positive relationship between firm size and leverage; the pecking order theory is too in favour of a positive relationship between firm size and debt, based on lower information asymmetry in larger firms between insiders and outside investors (Mugoša, 2015; Pinková, 2012).

Regarding firm age, the trade-off theory assumes a positive relationship between age and leverage, as older firms 
usually have advantages in profitability and diversification and then show lower likelihood of financial distress compared to younger firms (Cole, 2013). By contrast, the expectation of the pecking order theory is unclear: on the one hand, older firms should accumulate more internally generated funds and then require less debt; on the other hand, older firms should have less problems of informational asymmetry and then be easier to obtain debt (Avarmaa, Hazak, \& Männasoo, 2011).

As for profitability, the pecking order theory supports a negative relationship between profitability and leverage, since the firms with higher profitability could have more internally generated cash flow (thus requiring less external financing); by contrast, according to the trade-off theory, there should be a positive relationship between profitability and leverage, because of the higher capacity to support more debt and less bankruptcy costs (Dasilas \& Papasyriopoulos, 2015; Pepur, Ćurak, \& Poposki, 2016).

In terms of growth, according to Lowe et al. (1994, p.249), "lenders lend on the basis of future expectations and management may accept restrictive debt conditions in return for leveraged growth". And, based on this, they suppose that the relationship between sales growth and leverage should be positive. In addition, a positive relationship between growth and leverage is also supported by the pecking order theory, because of the increase in requirement for external financial sources (Pinková, 2012). On the other hand, as pointed out by Cwynar et al. (2015), because the fast-growing firms may face with the threat of instability which means being risker, the trade-off theory assumes a negative relationship between growth and leverage.

Aside from the above firm-related factors (firm size, age, profitability, and growth), industry also works as an important influential factor on leverage. From theoretical perspective, Degryse et al. (2012) argue that, the trade-off theory supports the existence of the optimal leverage ratio as the target of the firms in an industry (which comes from industry fixed effects), whereas the pecking order theory shows no clear prediction about industry fixed effects. As stated by La Rocca et al. (2011), the firms in the same industry should face similar circumstances, and then should show similar financing choices. From the perspective of empirical studies, Arce et al. (2015) find that industry mean or median capital structure does impact on the change of firm's capital structures.

In addition to the above mentioned theoretical and empirical studies that build the framework for researching on capital structure and its influential factors, there are some researchers focusing on the firms in some specific industry sectors and in some specific countries. For instance, the research paper of Matias et al. (2018) is about the capital structure of hotel firms in Portugal; the study of Jędrzejczak-Gas (2018) is regarding the determinants of the capital structure of enterprises in transport, spedition, and logistics sector in Poland; and the article of Schäfer et al. (2004) is about the determinants of debt and equity financing of young high-tech SMEs in Germany.

\section{Data, Variables, and Methodology}

This paper chooses the small and medium-sized enterprises (SMEs) in the high technology and medium-high technology manufacturing sectors in Portugal from the Iberian Balance Sheet Analysis System (SABI; developed by Bureau Van Dijk) database to build the total sample. Here, according to the high-tech classification of manufacturing industries based on NACE Rev. 2 2-digit level code (from Eurostat), high technology manufacturing sectors include manufacture of basic pharmaceutical products and pharmaceutical preparations (21) and manufacture of computer, electronic and optical products (26); while medium-high technology manufacturing sectors contain manufacture of chemicals and chemical products (20), manufacture of electrical equipment (27), manufacture of machinery and equipment n.e.c. (28), manufacture of motor vehicles, trailers and semi-trailers (29), and manufacture of other transport equipment (30).

Following the research of García-Teruel and Martínez-Solano (2007) where the criteria for defining SME include all the restrictions on the number of employee, turnover, and assets, we classify SME (with referring to the criteria of European Union) as: number of employees less than 250; turnover less than or equal to 50 million Euros; and balance sheet total less than or equal to 43 million Euros. The SMEs with missing data, outliers and errors are excluded, and we also require that in the whole studying period the sampled SMEs must show data in all the required variables, show leverage being less than 100 percent, and show positive operating revenues (referring to the sample-selecting standard of Cassar and Holmes (2003)). Finally, 1529 firms comprise the total sample with the required data from 2012 to 2016 (together with 2011 for calculating the growth between 2011 and 2012), and all the sampled firms are from high and medium-high technology manufacturing sectors. What is more, in order to study the capital structure in depth, the total sample is further categorized into young SMEs and mature SMEs based on the classification standard of Haltiwanger et al. (2013), where young firms are those no more than 10 years old and mature firms are those beyond 10 years old. 
In this paper, we choose total debt ratio, short-term debt ratio and long-term debt ratio as dependent variables, referring to the studies of Šarlija and Harc (2012) and Bolek and Lyroudi (2015) where the impacts on short-term and long-term leverage are also researched together with the total leverage when studying capital structure. As for the independent variables, we choose the variables to reflect profitability, growth, asset structure, size, age, and industry sector. In particular, we use intangible assets divided by assets as the proxy of growth opportunities (Acedo-Ramírez et al., 2013), tangible assets (tangible fixed assets and inventories) divided by total assets as the proxy of asset structure (Sogorb-Mira, 2005), and the natural logarithm of total assets as the proxy of size (Serghiescu \& Văidean, 2014). The details of the definitions of variables are shown in Table 1.

Table 1. Definitions of variables

\begin{tabular}{ll}
\hline Dependent variables & Measurements \\
\hline Total debt ratio (Leverage) & $\begin{array}{l}\text { Indebtedness: (Total shareholders' funds and liabilities - Shareholders' } \\
\text { equity)/Total shareholders funds and liabilities }\end{array}$ \\
\hline Long-term debt ratio & Non-current liabilities/Total assets \\
\hline Short-term debt ratio & Current liabilities/Total assets \\
\hline Independent variables & Measurements \\
\hline Firm size & Natural logarithm of total assets: Ln total assets in thousands of Euros \\
\hline Profitability (ROA) & Economic profitability: Profits before tax/Total assets \\
\hline Intangible assets (Intangibles) & Intangible fixed assets/Total assets \\
\hline Asset structure (Tangibles) & (Tangible fixed assets + Inventories)/Total assets \\
\hline Growth & $\begin{array}{l}\text { (The operating revenues in year } \mathrm{t}-\text { The operating revenues in year } \\
\text { t-1)/The operating revenues in year t-1 }\end{array}$ \\
\hline Firm age & $\begin{array}{l}\text { Age since the incorporation year to 2012, which changes with the } \\
\text { increase in year. }\end{array}$ \\
\hline Industry sectors & $\begin{array}{l}\text { NACE Rev. 2 (2-digit level code): 20, 21, 26, 27, 28, 29, 30 (With seven } \\
\text { industry sectors, six industry dummy variables are generated.) }\end{array}$ \\
\hline
\end{tabular}

The balanced panel model is used in this paper for studying capital structure, which is also employed in the research of Daskalakis and Psillaki (2008); they also enumerate some advantages of the panel data model, such as, reducing collinearity among the explanatory variables, including the heterogeneity that characterizes firms, and allowing dynamic effects. Similar to the study of Serghiescu and Văidean (2014) where both the fixed effects model and the ordinary least squares method are used for the balanced panel data, in addition to the fixed effects model, the pooled regression model is also introduced as the robustness test and for adding industry sector dummy in. Therefore, the next section firstly illustrates the results of the fixed effects model and the pooled regression model and then summarize the common results of the two models. 


\section{Results and Discussion}

4.1 The Results of the Fixed Effects Model (Tables 2, 3, and 4)

Table 2. The results of the fixed effects model on total leverage

\begin{tabular}{|c|c|c|c|c|c|c|}
\hline \multicolumn{7}{|c|}{ Dependent variable: Total leverage } \\
\hline \multicolumn{3}{|c|}{ Total sample } & \multicolumn{2}{|l|}{ Young firms } & \multicolumn{2}{|l|}{ Mature firms } \\
\hline \multicolumn{3}{|l|}{ Number of firms: 1,529} & \multicolumn{2}{|l|}{403} & \multicolumn{2}{|l|}{1,126} \\
\hline \multicolumn{3}{|c|}{ Number of observations: 7,645} & \multicolumn{2}{|l|}{2,015} & \multicolumn{2}{|l|}{5,630} \\
\hline \multicolumn{3}{|c|}{ R-squares within $=0.1691$} & \multicolumn{2}{|l|}{0.3298} & \multicolumn{2}{|l|}{0.1198} \\
\hline \multicolumn{3}{|c|}{ R-squares between $=0.0584$} & \multicolumn{2}{|l|}{0.0738} & \multicolumn{2}{|l|}{0.0277} \\
\hline \multicolumn{3}{|c|}{ R-squares overall $=0.0594$} & \multicolumn{2}{|l|}{0.0923} & \multicolumn{2}{|l|}{0.0295} \\
\hline \multicolumn{3}{|c|}{$\mathrm{F}(6,6110)=207.29$} & \multicolumn{2}{|c|}{$\mathrm{F}(6,1606)=131.71$} & \multicolumn{2}{|c|}{$\mathrm{F}(6,4498)=102.08$} \\
\hline \multicolumn{3}{|l|}{ Prob $>F=0.0000$} & \multicolumn{2}{|c|}{ Prob $>F=0.0000$} & \multicolumn{2}{|c|}{ Prob $>F=0.0000$} \\
\hline \multicolumn{3}{|l|}{ corr $\left(\mathrm{u} \_\mathrm{i}, \mathrm{Xb}\right)=-0.6605$} & \multicolumn{2}{|l|}{-0.5313} & \multicolumn{2}{|l|}{-0.5335} \\
\hline \multicolumn{3}{|c|}{ F test: $F(1528,6110)=31.09$} & \multicolumn{2}{|c|}{$F(402,1606)=23.02$} & \multicolumn{2}{|c|}{$\mathrm{F}(1125,4498)=35.89$} \\
\hline \multicolumn{3}{|c|}{ Prob $>F=0.0000$} & \multicolumn{2}{|c|}{ Prob $>\mathrm{F}=0.0000$} & \multicolumn{2}{|c|}{ Prob $>F=0.0000$} \\
\hline \multicolumn{3}{|c|}{$\operatorname{chi} 2(6)=(b-B)^{\prime}\left[\left(\mathrm{V} \_b-V \_B\right)^{\wedge}(-1)\right](b-B)=469.29$} & $\operatorname{chi} 2(6)=189$ & & $\operatorname{chi} 2(6)=213$ & \\
\hline Prob $>$ chi $2=0.0000$ & & & Prob $>\operatorname{chi} 2=$ & 0.0000 & Prob $>$ chi $2=$ & 0.0000 \\
\hline Independent variables & Coefficient & $\mathrm{P}>|\mathrm{t}|$ & Coefficient & $\mathrm{P}>|\mathrm{t}|$ & Coefficient & $\mathrm{P}>|\mathrm{t}|$ \\
\hline Ln assets & $0.087^{*}$ & 0.000 & $0.127^{*}$ & 0.000 & $0.072^{*}$ & 0.000 \\
\hline ROA & $-0.240^{*}$ & 0.000 & $-0.340^{*}$ & 0.000 & $-0.198^{*}$ & 0.000 \\
\hline Intangibles & $0.199^{*}$ & 0.004 & $0.266^{*}$ & 0.003 & 0.114 & 0.344 \\
\hline Tangibles & $0.022^{*}$ & 0.062 & -0.001 & 0.955 & $0.032^{*}$ & 0.026 \\
\hline Growth & 0.000045 & 0.507 & 0.000014 & 0.855 & $0.012^{*}$ & 0.000 \\
\hline Age & $-0.017 *$ & 0.000 & $-0.035^{*}$ & 0.000 & $-0.012^{*}$ & 0.000 \\
\hline Constant & $0.326^{*}$ & 0.000 & $0.100^{*}$ & 0.008 & $0.332^{*}$ & 0.000 \\
\hline
\end{tabular}

Note: “*” means being statistically significant at least at the 0.1 level.

Table 3. The results of the fixed effects model on short-term debt

\begin{tabular}{|c|c|c|c|c|c|c|}
\hline \multicolumn{7}{|c|}{ Dependent variable: Shot-term debt ratio } \\
\hline \multicolumn{3}{|l|}{ Total sample } & \multicolumn{2}{|l|}{ Young firms } & \multicolumn{2}{|c|}{ Mature firms } \\
\hline \multicolumn{3}{|l|}{ Number of firms: 1,529} & \multicolumn{2}{|l|}{403} & \multicolumn{2}{|c|}{1,126} \\
\hline \multicolumn{3}{|c|}{ Number of observation: 7,645} & \multicolumn{2}{|l|}{2,015} & \multicolumn{2}{|l|}{5,630} \\
\hline \multicolumn{3}{|c|}{ R-squares within $=0.0641$} & \multicolumn{2}{|l|}{0.1583} & \multicolumn{2}{|l|}{0.0422} \\
\hline \multicolumn{3}{|c|}{ R-squares between $=0.0299$} & \multicolumn{2}{|l|}{0.0521} & \multicolumn{2}{|l|}{0.0062} \\
\hline \multicolumn{3}{|c|}{ R-squares overall $=0.0276$} & \multicolumn{2}{|l|}{0.0657} & \multicolumn{2}{|l|}{0.0066} \\
\hline \multicolumn{3}{|c|}{$\mathrm{F}(6,6110)=69.69$} & \multicolumn{2}{|c|}{$\mathrm{F}(6,1606)=50.34$} & \multicolumn{2}{|c|}{$\mathrm{F}(6,4498)=33.03$} \\
\hline \multicolumn{3}{|l|}{ Prob $>F=0.0000$} & \multicolumn{2}{|c|}{ Prob $>F=0.0000$} & \multicolumn{2}{|c|}{ Prob $>F=0.0000$} \\
\hline \multicolumn{3}{|c|}{ corr $\left(\mathrm{u} \_\mathrm{i}, \mathrm{Xb}\right)=-0.6906$} & \multicolumn{2}{|c|}{-0.3368} & \multicolumn{2}{|c|}{-0.5825} \\
\hline \multicolumn{3}{|c|}{ F test: $F(1528,6110)=15.47$} & \multicolumn{2}{|c|}{$\mathrm{F}(402,1606)=12.51$} & \multicolumn{2}{|c|}{$\mathrm{F}(1125,4498)=16.98$} \\
\hline \multicolumn{3}{|l|}{ Prob $>F=0.0000$} & \multicolumn{2}{|c|}{ Prob $>F=0.0000$} & \multicolumn{2}{|c|}{ Prob $>F=0.0000$} \\
\hline \multicolumn{3}{|c|}{ chi2 $(6)=(b-B))^{\prime}\left[\left(V \_b-V \_B\right)^{\wedge}(-1)\right](b-B)=255.81$} & $\operatorname{chi} 2(6)=59$. & & $\operatorname{chi} 2(6)=13$ & \\
\hline Prob $>$ chi $2=0.0000$ & & & Prob $>$ chi $2=$ & 0.0000 & Prob $>$ chi $2=$ & 0.0000 \\
\hline Independent variables & Coefficient & $\mathrm{P}>|\mathrm{t}|$ & Coefficient & $\mathrm{P}>|\mathrm{t}|$ & Coefficient & $\mathrm{P}>|\mathrm{t}|$ \\
\hline Ln assets & $0.033^{*}$ & 0.000 & $0.073 *$ & 0.000 & $0.013 *$ & 0.064 \\
\hline ROA & $-0.132 *$ & 0.000 & $-0.231 *$ & 0.000 & $-0.089 *$ & 0.000 \\
\hline Intangibles & -0.119 & 0.169 & -0.145 & 0.220 & 0.012 & 0.936 \\
\hline Tangibles & $-0.139 *$ & 0.000 & $-0.259 *$ & 0.000 & $-0.064 *$ & 0.000 \\
\hline Growth & -0.000047 & 0.584 & -0.000087 & 0.388 & $0.013 *$ & 0.000 \\
\hline Age & $-0.015^{*}$ & 0.000 & $-0.030 *$ & 0.000 & $-0.011^{*}$ & 0.000 \\
\hline Constant & $0.554 *$ & 0.000 & $0.320 *$ & 0.000 & $0.601 *$ & 0.000 \\
\hline
\end{tabular}

Note: “*” means being statistically significant at least at the 0.1 level. 
Table 4. The results of the fixed effects model on long-term debt

\begin{tabular}{|c|c|c|c|c|c|c|}
\hline \multicolumn{7}{|c|}{ Dependent variable: Long-term debt ratio } \\
\hline \multicolumn{3}{|c|}{ Total sample } & \multicolumn{2}{|l|}{ Young firms } & \multicolumn{2}{|c|}{ Mature firms } \\
\hline \multicolumn{3}{|l|}{ Number of firms: 1,529} & \multicolumn{2}{|l|}{403} & \multicolumn{2}{|c|}{1,126} \\
\hline \multicolumn{3}{|c|}{ Number of observation: 7,645} & \multicolumn{2}{|l|}{2,015} & \multicolumn{2}{|l|}{5,630} \\
\hline \multicolumn{3}{|c|}{ R-squares within $=0.0625$} & \multicolumn{2}{|l|}{0.1112} & \multicolumn{2}{|l|}{0.0423} \\
\hline \multicolumn{3}{|c|}{ R-squares between $=0.0296$} & \multicolumn{2}{|l|}{0.0736} & \multicolumn{2}{|l|}{0.0067} \\
\hline \multicolumn{3}{|c|}{ R-squares overall $=0.0297$} & \multicolumn{2}{|l|}{0.0726} & \multicolumn{2}{|l|}{0.0076} \\
\hline \multicolumn{3}{|c|}{$\mathrm{F}(6,6110)=67.92$} & \multicolumn{2}{|c|}{$F(6,1606)=33.48$} & \multicolumn{2}{|c|}{$\mathrm{F}(6,4498)=33.11$} \\
\hline \multicolumn{3}{|l|}{ Prob > F = 0.0000} & \multicolumn{2}{|c|}{ Prob $>F=0.0000$} & \multicolumn{2}{|c|}{ Prob $>F=0.0000$} \\
\hline \multicolumn{3}{|c|}{ corr $\left(u \_i, X b\right)=-0.4292$} & \multicolumn{2}{|c|}{-0.4165} & \multicolumn{2}{|c|}{-0.4931} \\
\hline \multicolumn{3}{|c|}{$F$ test: $F(1528,6110)=10.71$} & \multicolumn{2}{|c|}{$\mathrm{F}(402,1606)=8.19$} & \multicolumn{2}{|c|}{$\mathrm{F}(1125,4498)=12.36$} \\
\hline \multicolumn{3}{|c|}{ Prob $>\mathrm{F}=0.0000$} & \multicolumn{2}{|c|}{ Prob $>F=0.0000$} & \multicolumn{2}{|c|}{ Prob $>F=0.0000$} \\
\hline \multicolumn{3}{|c|}{ chi2(5)=(b-B)'[(V_b-V_B $\left.)^{\wedge}(-1)\right](b-B)=174.72$} & $\operatorname{chi} 2(6)=111$ & & $\operatorname{chi} 2(6)=155$ & \\
\hline Prob>chi2 $=0.0000$ & & & Prob $>$ chi $2=$ & 0000 & Prob $>$ chi $2=$ & .0000 \\
\hline Independent variables & Coefficient & $\mathrm{P}>|\mathrm{t}|$ & Coefficient & $\mathrm{P}>|\mathrm{t}|$ & Coefficient & $\mathrm{P}>|\mathrm{t}|$ \\
\hline Ln assets & $0.054 *$ & 0.000 & $0.054^{*}$ & 0.000 & $0.059^{*}$ & 0.000 \\
\hline ROA & $-0.108 *$ & 0.000 & $-0.109 *$ & 0.000 & $-0.109 *$ & 0.000 \\
\hline Intangibles & $0.318^{*}$ & 0.000 & $0.411^{*}$ & 0.000 & 0.101 & 0.437 \\
\hline Tangibles & $0.161 *$ & 0.000 & $0.258^{*}$ & 0.000 & $0.096^{*}$ & 0.000 \\
\hline Growth & 0.000093 & 0.220 & 0.0001 & 0.272 & -0.001 & 0.546 \\
\hline Age & $-0.002^{*}$ & 0.005 & $-0.005^{*}$ & 0.010 & -0.001 & 0.113 \\
\hline Constant & $-0.228^{*}$ & 0.000 & $-0.220^{*}$ & 0.000 & $-0.269 *$ & 0.000 \\
\hline
\end{tabular}

Note: “*” means being statistically significant at least at the 0.1 level.

Here we set the statistically significant level at 0.1 , and in the following parts only the statistically significant variables are discussed. As for the results of the fixed effect model on the total leverage, total assets (with a positive relationship), ROA (with a negative relationship), and age (with a negative relationship) are statistically significant and keep stable relationships with total leverage in all the regressions of the total sample, the young group and the mature group. Both growth (in the mature group) and tangible assets (in the total sample and the mature group) are positively related to total leverage. Intangible assets show statistical significance in the regressions of the total sample and the young group, with stable positive effect.

Regarding short-term debt, total assets (with a positive relationship), ROA (with a negative relationship), age (with a negative relationship), and tangible assets (with a negative relationship) are statistically significant and keep stable relationships with short-term debt in all the regressions of the total sample, the young group and the mature group. Growth only shows statistical significance in the regression of mature group, with positive effect.

In terms of long-term debt, total assets (with a positive relationship), ROA (with a negative relationship), and tangible assets (with a positive relationship) are statistically significant and keep stable relationships with long-term debt in all the regressions of the total sample, the young group and the mature group. Intangible assets (with a positive relationship) and age (with a negative relationship) show statistical significance in the regressions of the total sample and the young group. 


\subsection{The Results of the Pooled Regression Model (Tables 5, 6, and 7)}

Table 5. The results of the pooled regressions on total leverage

Dependent variable: Total leverage

\begin{tabular}{|c|c|c|c|c|c|c|}
\hline \multirow{2}{*}{\multicolumn{3}{|c|}{$\begin{array}{l}\text { Total sample } \\
\text { Number of observations: } 7,645\end{array}$}} & \multicolumn{2}{|c|}{ Young Firms } & \multicolumn{2}{|c|}{ Mature Firms } \\
\hline & & & \multicolumn{2}{|l|}{2,015} & \multicolumn{2}{|c|}{5,630} \\
\hline \multicolumn{3}{|l|}{$F(12,7632)=116.39$} & \multicolumn{2}{|c|}{$F(12,2002)=47.56$} & \multicolumn{2}{|c|}{$F(12,5617)=67.42$} \\
\hline \multicolumn{3}{|l|}{ Prob $>F=0.0000$} & \multicolumn{2}{|c|}{ Prob $>F=0.0000$} & \multicolumn{2}{|c|}{ Prob $>F=0.0000$} \\
\hline \multicolumn{3}{|l|}{ R-squared: 0.1547} & \multicolumn{2}{|c|}{0.2218} & \multicolumn{2}{|l|}{0.1259} \\
\hline \multicolumn{3}{|c|}{ Adjusted R-squared: 0.1534} & 0.2172 & & 0.1240 & \\
\hline \multicolumn{3}{|c|}{ Root MSE $=0.22309$} & \multicolumn{2}{|l|}{0.21505} & \multicolumn{2}{|l|}{0.22174} \\
\hline Independent variables & Coefficient & $\mathrm{P}>|\mathrm{t}|$ & Coefficient & $\mathrm{P}>|\mathrm{t}|$ & Coefficient & $\mathrm{P}>|\mathrm{t}|$ \\
\hline Ln assets & $0.009 *$ & 0.000 & $0.021 *$ & 0.000 & $0.008 *$ & 0.000 \\
\hline ROA & $-0.397 *$ & 0.000 & $-0.445^{*}$ & 0.000 & $-0.402 *$ & 0.000 \\
\hline Intangibles & 0.068 & 0.324 & $-0.233^{*}$ & 0.009 & $0.365^{*}$ & 0.001 \\
\hline Tangibles & $0.235^{*}$ & 0.000 & $0.222 *$ & 0.000 & $0.236^{*}$ & 0.000 \\
\hline Growth & $0.0003219 *$ & 0.045 & 0.0002544 & 0.104 & $0.028 *$ & 0.000 \\
\hline Age & $-0.004 *$ & 0.000 & $-0.017 *$ & 0.000 & $-0.003 *$ & 0.000 \\
\hline Industry dummy 1 & $0.030^{*}$ & 0.034 & $0.073 *$ & 0.003 & 0.013 & 0.472 \\
\hline Industry dummy 2 & $0.064 *$ & 0.001 & $0.074 *$ & 0.046 & $0.049 *$ & 0.029 \\
\hline Industry dummy 3 & -0.008 & 0.653 & 0.0000395 & 0.999 & -0.003 & 0.874 \\
\hline Industry dummy 4 & 0.010 & 0.496 & $0.067 *$ & 0.007 & -0.009 & 0.620 \\
\hline Industry dummy 5 & 0.020 & 0.147 & $0.079 *$ & 0.001 & 0.000659 & 0.969 \\
\hline Industry dummy 6 & $0.030^{*}$ & 0.050 & $0.075^{*}$ & 0.005 & 0.017 & 0.345 \\
\hline Constant & $0.452 *$ & 0.000 & $0.472 *$ & 0.000 & $0.426^{*}$ & 0.000 \\
\hline
\end{tabular}

Note: "*” means being statistically significant at least at the 0.1 level.

Table 6 . The results of the pooled regressions on short-term debt

Dependent variable: Short-term debt ratio

\begin{tabular}{|c|c|c|c|c|c|c|}
\hline \multirow{2}{*}{\multicolumn{3}{|c|}{$\begin{array}{l}\text { Total Sample } \\
\text { Number of observations: } 7,645\end{array}$}} & \multicolumn{2}{|c|}{ Young Firms } & \multicolumn{2}{|c|}{ Mature Firms } \\
\hline & & & \multicolumn{2}{|l|}{2,015} & \multicolumn{2}{|c|}{5,630} \\
\hline \multicolumn{3}{|l|}{$\mathrm{F}(12,7632)=35.12$} & \multicolumn{2}{|c|}{$\mathrm{F}(12,2002)=22.42$} & \multicolumn{2}{|c|}{$F(12,5617)=17.13$} \\
\hline \multicolumn{3}{|l|}{ Prob > F $=0.0000$} & \multicolumn{2}{|c|}{ Prob $>\mathrm{F}=0.0000$} & \multicolumn{2}{|c|}{ Prob $>\mathrm{F}=0.0000$} \\
\hline \multicolumn{3}{|l|}{ R-squared: 0.0523} & \multicolumn{2}{|c|}{0.1185} & \multicolumn{2}{|c|}{0.0353} \\
\hline \multicolumn{3}{|c|}{ Adjusted R-squared: 0.0508} & 0.1132 & & 0.0332 & \\
\hline \multicolumn{3}{|c|}{ Root MSE $=0.21123$} & \multicolumn{2}{|l|}{0.22226} & \multicolumn{2}{|l|}{0.20243} \\
\hline Independent variables & Coefficient & $\mathrm{P}>|\mathrm{t}|$ & Coefficient & $P>|t|$ & Coefficient & $\mathrm{P}>|\mathrm{t}|$ \\
\hline Ln assets & $0.015^{*}$ & 0.000 & $0.028^{*}$ & 0.000 & $0.013 *$ & 0.000 \\
\hline ROA & $-0.255^{*}$ & 0.000 & $-0.319^{*}$ & 0.000 & $-0.250 *$ & 0.000 \\
\hline Intangibles & $-0.210^{*}$ & 0.001 & $-0.382^{*}$ & 0.000 & -0.080 & 0.409 \\
\hline Tangibles & -0.003 & 0.779 & $-0.069 *$ & 0.002 & 0.017 & 0.167 \\
\hline Growth & -0.000052 & 0.735 & -0.000164 & 0.309 & $0.023 *$ & 0.000 \\
\hline Age & $-0.003 *$ & 0.000 & $-0.017 *$ & 0.000 & $-0.002 *$ & 0.000 \\
\hline Industry dummy 1 & $0.039 *$ & 0.004 & $0.132 *$ & 0.000 & -0.000994 & 0.950 \\
\hline Industry dummy 2 & $0.041^{*}$ & 0.025 & 0.063 & 0.104 & 0.016 & 0.451 \\
\hline
\end{tabular}




\begin{tabular}{lllllll}
\hline Industry dummy 3 & 0.006 & 0.699 & $0.061^{*}$ & 0.027 & -0.014 & 0.471 \\
\hline Industry dummy 4 & 0.016 & 0.262 & $0.113^{*}$ & 0.000 & -0.025 & 0.129 \\
\hline Industry dummy 5 & 0.018 & 0.161 & $0.112^{*}$ & 0.000 & -0.020 & 0.204 \\
\hline Industry dummy 6 & $0.032^{*}$ & 0.026 & $0.136^{*}$ & 0.000 & -0.005 & 0.771 \\
\hline Constant & $0.327^{*}$ & 0.000 & $0.324^{*}$ & 0.000 & $0.318^{*}$ & 0.000 \\
\hline
\end{tabular}

Note: “*” means being statistically significant at least at the 0.1 level.

Table 7. The results of the pooled regressions on long-term debt

Dependent variable: Long-term debt ratio

\begin{tabular}{|c|c|c|c|c|c|c|}
\hline \multirow{2}{*}{\multicolumn{3}{|c|}{$\begin{array}{l}\text { Total Sample } \\
\text { Number of observations: } 7,645\end{array}$}} & \multicolumn{2}{|c|}{ Young Firms } & \multicolumn{2}{|c|}{ Mature Firms } \\
\hline & & & \multicolumn{2}{|l|}{2,015} & \multicolumn{2}{|c|}{5,630} \\
\hline \multicolumn{3}{|c|}{$\mathrm{F}(12,7632)=100.26$} & \multicolumn{2}{|c|}{$F(12,2002)=32.49$} & \multicolumn{2}{|c|}{$F(12,5617)=67.72$} \\
\hline \multicolumn{3}{|l|}{ Prob > F = 0.0000} & \multicolumn{2}{|c|}{ Prob $>F=0.0000$} & \multicolumn{2}{|c|}{ Prob $>F=0.0000$} \\
\hline \multicolumn{3}{|l|}{ R-squared: 0.1362} & \multicolumn{2}{|c|}{0.1630} & \multicolumn{2}{|l|}{0.1264} \\
\hline \multicolumn{3}{|c|}{ Adjusted R-squared: 0.1348} & 0.1580 & & 0.1245 & \\
\hline \multicolumn{3}{|c|}{ Root MSE: 0.160} & \multicolumn{2}{|l|}{0.175} & \multicolumn{2}{|l|}{0.154} \\
\hline Independent variables & Coefficient & $\mathrm{P}>|\mathrm{t}|$ & Coefficient & $\mathrm{P}>|\mathrm{t}|$ & Coefficient & $\mathrm{P}>|\mathrm{t}|$ \\
\hline Ln assets & $-0.006^{*}$ & 0.000 & $-0.008^{*}$ & 0.009 & $-0.005^{*}$ & 0.000 \\
\hline ROA & $-0.142 *$ & 0.000 & $-0.126^{*}$ & 0.000 & $-0.151^{*}$ & 0.000 \\
\hline Intangibles & $0.278^{*}$ & 0.000 & $0.149^{*}$ & 0.041 & $0.444^{*}$ & 0.000 \\
\hline Tangibles & $0.238 *$ & 0.000 & $0.291 *$ & 0.000 & $0.219 *$ & 0.000 \\
\hline Growth & $0.000374 *$ & 0.001 & $0.000419 *$ & 0.001 & 0.005 & 0.151 \\
\hline Age & $-0.001 *$ & 0.000 & 0.000391 & 0.751 & $-0.001 *$ & 0.000 \\
\hline Industry dummy 1 & -0.008 & 0.418 & $-0.059 *$ & 0.003 & 0.014 & 0.265 \\
\hline Industry dummy 2 & $0.023 *$ & 0.092 & 0.012 & 0.696 & $0.034 *$ & 0.032 \\
\hline Industry dummy 3 & -0.014 & 0.256 & $-0.061 *$ & 0.005 & 0.011 & 0.474 \\
\hline Industry dummy 4 & -0.006 & 0.595 & $-0.046^{*}$ & 0.021 & 0.016 & 0.201 \\
\hline Industry dummy 5 & 0.002 & 0.863 & $-0.033^{*}$ & 0.080 & $0.020^{*}$ & 0.085 \\
\hline Industry dummy 6 & -0.002 & 0.832 & $-0.061 *$ & 0.004 & $0.022 *$ & 0.082 \\
\hline Constant & $0.124 *$ & 0.000 & $0.148 *$ & 0.000 & $0.107^{*}$ & 0.000 \\
\hline
\end{tabular}

Note: “*” means being statistically significant at least at the 0.1 level;

As for the results of the pooled regression model on total leverage, the statistically significant variables with stable relationships with total leverage in all the regressions of the total sample, the young group and the mature group include total assets (being positively related to leverage), ROA (being negatively related to leverage), tangible assets (being positively related to leverage), and age (being negative related to leverage). Growth is statistically significant and positively related to total leverage in the regressions of the total sample and the mature group. Instable relationship to total leverage is observed for intangible assets when being statistically significant in the regressions of the young group (with a negative relationship) and the mature group (with a positive relationship). Industry dummy is observed to be statistically significant in all the three groups (the total sample, the young group and the mature group).

In terms of short-term debt, statistically significant variables with stable relationships with short-term debt in all the regressions of the total sample, the young group and the mature group contain total assets (being positively related to short-term debt), ROA (being negatively related to short-term debt), and age (being negatively related to short-term debt). Growth is statistically significant and positively related to short-term debt in the regression of the mature group. Intangible assets and tangible assets are negatively related to short-term debt when being statistical significant (in the total sample and the young group for intangible assets and in the young group for tangible assets). Industry dummy is observed to be statistically significant in all the groups, except for the mature group. 
Regarding long-term debt, statistically significant variables with stable relationships with long-term debt in all the regressions of the total sample, the young group and the mature group include total assets (being negatively related to long-term debt), ROA (being negatively related to long-term debt), tangible assets (being positively related to long-term debt), and intangible assets (being positively related to long-term debt). Growth is positively related to long-term debt when being statistically significant in the regressions of the total sample and the young group, while age shows a statistically significant negative relationship with long-term debt in the regressions of the total sample and the mature group. Industry dummy is observed to be statistically significant in all the groups.

\subsection{Discussion}

Here, albeit not totally shown in the tables, all the results of F-test (Prob $>F=0.0000$ ) show that the fixed effects model is better than the pooled regression model, and all the results of Hausman-test (Prob>chi2 $=0.0000$ ) illustrate that the fixed effects model is better than the random model. So there is no need to do Breusch-Pagan test to compare the random model with the pooled regression model. Besides, with creating the cross variables between the independent variables and the dummy variable of young and mature firms, the results of Chow test support the statistically significant difference between the young group and the mature group.

Total assets are positively related to total leverage, short-term debt, and long-term debt in majority, though in the pooled regression model the relationship between total assets and long-term debt is negative. The positive relationship between firm size and leverage is also supported by most empirical evidence (Avarmaa et al., 2011). ROA is negatively related to total leverage, short-term debt, and long-term debt in all the regressions. The negative relationship between profitability and leverage corresponds to the research result of Fattouh et al. (2008) and Bartoloni (2013), who point out that the positive relationship between profitability and internal finance (or the negative relationship between profitability and debt ratio) indicates the pecking order mechanism. This is especially true for small firms which show more problems of informational opacity and then rely more on internal funds (Jõeveer, 2013).

Age is negatively related to total leverage, short-term debt, and long-term debt in most regressions. The negative relationship between firm age and leverage here is consistent with the results of most empirical studies, as pointed out by Van Caneghem and Van Campenhout (2012); they interpret this negative relationship as younger firms being poor in generating profits, thus requiring external debts. From the theory point of view, the negative relationships between age and debt-related variables tend to partially support the pecking order theory about the advantages of older firms in generating retained earnings (Cole, 2013).

The impact of intangible assets on the debt variables is instable: the results here partially support the findings of Sogorb-Mira (2005) (that is, a positive relationship between growth opportunities represented by the ratio of intangible assets to total assets and firm leverage but a negative relationship between growth opportunities and short-term debt) especially in the total sample and young group; and the positive effect of intangible assets on long-term debt seems to be strong, which is similar to the finding of Mateev and Ivanov (2011). The instable effects to some extent may be related to the heterogeneity of intangible assets, because for different types of intangible assets firms may have different financing policies (Alves \& Martins, 2009). And, more importantly as pointed out by Chappell and Jaffe (2018), intangibles tend to take long-run (rather than short-run) effects, which are shown here as the negative relationship with short-term debt and positive relationship with long-term debt.

Tangible assets also do not show stable relationship with the debt variables; nonetheless, similar to the findings of Koralun-Bereźnicka (2013), its positive effect on long-term debt and negative effect on short-term debt tend to be clear. Theoretically, as pointed out by Fattouh et al. (2008), more tangible assets mean more collateralisable assets, which can lead to higher leverage; however, a negative relationship between asset tangibility and leverage may also exist, as the firms with large amount of tangible assets (especially fixed assets) do not need to invest much and then require less borrowing (Arsov \& Naumoski, 2016). The results here are to some extent correspond to the statement of Harc (2015)-that is, because of the effect of collateral (as an important function of tangible assets) on reducing risk, SMEs tend to use collateral for attracting long-term debt, which would cause negative impact of collateral on short-term debt and then positive impact on total debt contributed by long-term debt.

Growth shows a positive relationship with the debt variables, especially in the mature group with the total leverage and short-term debt. The results confirm that internal finance usually is not enough for the fast-growing firms (Mac an Bhaird \& Lucey, 2010), thus requiring external finance; this is in accord with the pecking order theory (Pinková, 2012). Industry sector is a statistically significant impacting factor (albeit not in all the regressions), which is also found in the research of (for example) Bauer (2004). Furthermore, it seems that, compared to mature SMEs, young SMEs tend to be impacted more by industry factor. 


\section{Conclusions}

To sum up, the results of this paper show that: firm size (total assets), profitability (ROA), and firm age are strong influential factors, as they show statistical significance (at the level of 0.1) in most regressions and tend to keep stable relationships with debt ratios; industry sector is also a statistically significant variable; tangible assets, intangible assets, and especially growth are relatively weak influential factors, because they do not show statistical significance as frequently as the strong ones do. Furthermore, the differences in the impacts on the capital structure between the young and mature groups are mainly shown by intangible assets, growth and industry sector. Hence, the results partially are in favour of the statement of La Rocca et al. (2011) about the differences in firm's capital structure drivers and financing strategies at the earlier stages and at other stages of their life cycles, which are caused by the different characteristics (such as asymmetric information and growth opportunities) at different stages.

Here, the generally positive effect of firm size, the negative effect of profitability, and the negative effect of age tend to give countenance to the pecking order theory, on the basis of less problems of informational asymmetry for the larger firms, more retained earnings for the more profitable firms relying less on external debts, and the higher capacity of self-funding and lower funding requirement for the older firms (Malinić, Denčić-Mihajlov, \& Ljubenović, 2013; Matias et al., 2018). On the other hand, the finding that industry sector works as a significant influential factor tends to support the trade-off theory about target capital structures in industries (Degryse et al., 2012); and the result that industry sector shows less influence on mature SMEs than it does on young SMEs may be interpreted as completion in capital structure adjustment for mature firms.

Because the studying target of this paper is the capital structure of Portuguese high technology and medium-high technology SMEs, it is necessary to pay special attention to intangible assets (considering the importance of intangible assets to this type of firms). In particular, intangible assets show more statistical significance in the young SME group compared to the mature SME group. From theoretical perspective, the instable relationships between intangible assets and debt variables may mirror the conflict between the argument of the trade-off theory about large financial distress costs for the firms with large amount of intangible assets and the argument of the pecking order theory about the requirement for not only the internal but also the external funds of the firms with growth opportunities represented by intangible asset (Mateev \& Ivanov, 2011); from the perspective of practice, the instable relationships also show the important impacts of not only the level but also the type of intangible assets on financial policy (Alves \& Martins, 2009). Besides, intangible assets tend to be positively related to long-term debt especially in young group, which may reflect the positive attitude of financial institutions on the value of intangibles in generating future benefits for young firms (because young firms should be easier to use new technology for generating intangible assets).

As for tangible assets, the differences in the results between the young group and the mature group are not as large as they are for intangible assets; to put it another way, there are more similarities. In concrete, tangible assets are positively related to long-term debt and total leverage, but negatively related to short-term debt. Here the positive relationships tend to support the trade-off theory and the pecking order theory, both of which (based on the collateral value of tangible assets) predict positive effects of tangible assets on increasing debt (Koralun-Bereźnicka, 2013). Furthermore, as stated by Harc (2015), SMEs tend to use collateral to attract long-term debt, thus leaving less collateral for short-term debt.

Growth is positively related to the debt ratios, especially in the mature group to total leverage and short-term debt. The results here to some extent support the pecking order theory, according to which rapid growth may cause internally generated funds being insufficient to finance growth, thus relying more on external financing (Acedo-Ramírez et al. 2013). Because the fast-growing firms may show more instability and be risker (Cwynar et al., 2015), creditors tend to lend short-term debt (instead of long-term debt) to these firms in order to reduce potential risk (Pindado et al. 2006).

All in all, this paper finds that firm size, profitability, firm age, and industry sector impact much on the capital structure and debt ratios of Portuguese high technology and medium-high technology SMEs. By contrast, the impacts of tangible assets, intangible assets, and growth are not as strong as the previous factors, and there exists great difference when intangible assets, growth, and industry sector work as influential factors separately for the young and mature SMEs. Therefore, following the research trend on studying capital structure in specific industries and in specific countries, this paper contributes to the empirical research on capital structure in high technology and medium-high technology sectors in Portugal. In addition, although the trade-off theory is supported by the results of some factors (for example industry sector), the results of most strong influential factors (such as firm size, age and profitability) tend to give countenance to the pecking order theory; thus further highlighting the influence of 
information asymmetry on high and medium-high technology SMEs. In spite of the small number of independent variables chosen here, the findings of this paper illustrate the important role of the pecking order theory in high and medium-high technology SMEs; so future research can continue to enrich this traditional topic with more evidence in specific industries and in specific countries.

\section{References}

Acedo-Ramírez, M. A., Ayala-Calvo, J. C., \& Rodríguez-Osés, J. E. (2013). Capital structure of small companies in the Spanish footwear sector: Relevant factors. SERIEs. Journal of the Spanish Economic Association, 4(2), 155-173. https://doi.org/10.1007/s13209-012-0085-8

Aghion, P., Bond, S., Klemm, A., \& Marinescu, I. (2004). Technology and financial structure: Are innovative firms different?. Journal of the European Economic Association, 2(2-3), 277-288. https://doi.org/10.1162/154247604323067989

Alves, S., \& Martins, J. (2009). The impact of intangible assets on financial and governance policies: A univariate analysis. Corporate Ownership and Control, 7(1), 416-433.

Andreeva, G., Calabrese, R., \& Osmetti, S. A. (2016). A comparative analysis of the UK and Italian small businesses using Generalised Extreme Value models. European Journal of Operational Research, 249(2), 506-516. https://doi.org/10.1016/j.ejor.2015.07.062

Arce, D. G., Cook, D. O., \& Kieschnick, R. L. (2015). On the evolution of corporate capital structures. Journal of Evolutionary Economics, 25(3), 561-583. https://doi.org/10.1007/s00191-015-0394-8

Arsov, S., \& Naumoski, A. (2016). Determinants of capital structure: An empirical study of companies from selected post-transition economies. Zbornik radova Ekonomskog fakulteta u Rijeci: časopis za ekonomsku teoriju i praksu - Proceedings of Rijeka Faculty of Economics: Journal of Economics and Business, 34(1), 119-146. https://doi.org/10.18045/zbefri.2016.1.119

Avarmaa, M., Hazak, A., \& Männasoo, K. (2011). Capital structure formation in multinational and local companies in the Baltic States. Baltic Journal of Economics, 11(1), 125-145.

Balakrishnan, S., \& Fox, I. (1993). Asset specificity, firm heterogeneity and capital structure. Strategic Management Journal, 14(1), 3-16.

Bartoloni, E. (2013). Capital structure and innovation: Causality and determinants. Empirica, 40(1), 111-151. https://doi.org/10.1007/s10663-011-9179-y

Bauer, P. (2004). Determinants of capital structure: Empirical evidence from the Czech Republic. Finance a úvěr-Czech Journal of Economics and Finance, 54(1-2), 2-21.

Bolek, M., \& Lyroudi, K. (2015). Is there any relation between intellectual capital and the capital structure of a company? The case of polish listed companies. Financial Internet Quarterly, e-Finanse, 11(4), 23-33. https://doi.org/10.14636/1734-039X_11_4_002

Bubic, J., \& Susak, T. (2016). Detecting optimal financial and capital structure: The case of small and medium enterprises (SME) in Republic of Croatia. Journal of Economic and Social Development, 3(1), 104-113.

Bukalska, E. (2019). Testing trade-off theory and pecking order theory under managerial overconfidence. International Journal of Management and Economics, 55(2), 99-117. https://doi.org/10.2478/ijme-2019-0008

Cassar, G., \& Holmes, S. (2003). Capital structure and financing of SMEs: Australian evidence. Accounting \& Finance, 43(2), 123-147. https://doi.org/10.1111/1467-629X.t01-1-00085

Chappell, N., \& Jaffe, A. (2018). Intangible investment and firm performance. Review of Industrial Organization, 52(4), 509-559. https://doi.org/10.1007/s11151-018-9629-9

Cole, R. A. (2013). What do we know about the capital structure of privately held US firms? Evidence from the surveys of small business finance. Financial Management, 42(4), 777-813. https://doi.org/10.1111/fima.12015

Cwynar, A., Cwynar, W., \& Dankiewicz, R., (2015). Studies of firm capital structure determinants in Poland: An integrative review. Financial Internet Quarterly e-Finanse, 11(4), 1-22. https://doi.org/10.14636/1734-039X_11_4_001

Dasilas, A., \& Papasyriopoulos, N. (2015). Corporate governance, credit ratings and the capital structure of Greek SME and large listed firms. Small Business Economics, 45(1), 215-244. https://doi.org/10.1007/s11187-015-9648-y 
Daskalakis, N., \& Psillaki, M. (2008). Do country or firm factors explain capital structure? Evidence from SMEs in France and Greece. Applied Financial Economics, 18(2), 87-97. https://doi.org/10.1080/09603100601018864

Degryse, H., de Goeij, P., \& Kappert, P. (2012). The impact of firm and industry characteristics on small firms' capital structure. Small Business Economics, 38(4), 431-447. https://doi.org/10.1007/s11187-010-9281-8

Fattouh, B., Harris, L., \& Scaramozzino, P. (2008). Non-linearity in the determinants of capital structure: Evidence from UK firms. Empirical Economics, 34(3), 417-438. https://doi.org/10.1007/s00181-007-0128-3

García-Teruel, P. J., \& Martínez-Solano, P. (2007). Short-term debt in Spanish SMEs. International Small Business Journal, 25(6), 579-602. https://doi.org/10.1177/0266242607082523

Haltiwanger, J., Jarmin, R. S., \& Miranda, J. (2013). Who creates jobs? Small versus large versus young. The Review of Economics and Statistics, 95(2), 347-361. https://doi.org/10.1162/REST_a_00288

Harc, M. (2015). The relationship between tangible assets and capital structure of small and medium-sized companies in Croatia. Ekonomski Vjesnik / Econviews-Review of Contemporary Business, Entrepreneurship and Economic Issues, 28(1), 213-224.

Horváthová, J., \& Mokrišová, M. (2017). Capital structure modelling and analysis of its impact on business performance. Financial Assets and Investing, 8(2), 19-36. https://doi.org/10.5817/FAI2017-2-2

Jędrzejczak-Gas, J. (2018). Determinants of the capital structure of TSL sector enterprises. Management, 22(1), 122-139. https://doi.org/10.2478/manment-2018-0013

Jensen, M. C., \& Meckling, W. H. (1976). Theory of the firm: Managerial behavior, agency costs and ownership structure. Journal of Financial Economics, 3(4), 305-360. https://doi.org/10.1016/0304-405X(76)90026-X

Jõeveer, K. (2013). What do we know about the capital structure of small firms?. Small Business Economics, 41(2), 479-501. https://doi.org/10.1007/s11187-012-9440-1

Koralun-Bereźnicka, J. (2013). How does asset structure correlate with capital structure? - Cross-industry and cross-size analysis of the EU countries. Universal Journal of Accounting and Finance, 1(1), 19-28. https://doi.org/10.13189/ujaf.2013.010103

La Rocca, M., La Rocca, T., \& Cariola, A. (2011). Capital structure decisions during a firm's life cycle. Small Business Economics, 37(1), 107-130. https://doi.org/10.1007/s11187-009-9229-z

Lowe, J., Naughton, T., \& Taylor, P. (1994). The impact of corporate strategy on the capital structure of Australian companies. Managerial and Decision Economics, 15(3), 245-257. https://doi.org/10.1002/mde.4090150306

Mac an Bhaird, C., \& Lucey, B. M. (2010). Determinants of capital structure in Irish SMEs. Small Business Economics, 35(3), 357-375. https://doi.org/10.1007/s11187-008-9162-6

Malinić, D., Denčić-Mihajlov, K., \& Ljubenović, E. (2013). The Determinants of Capital Structure in Emerging Capital Markets: Evidence from Serbia. European Research Studies, 16(2), 98-119.

Mateev, M., \& Ivanov, K. (2011). How SME uniqueness affects capital structure: Evidence from Central and Eastern Europe panel data. Quarterly Journal of Finance and Accounting, 50(1), 115-143.

Matias, F., Salsa, L., \& Afonso, C. M. (2018). Capital structure of Portuguese hotel firms: A structural equation modelling approach. Tourism \& Management Studies, 14(SI1), 73-82. http://dx.doi.org/10.18089/tms.2018.14SI108

Mihaela, H., \& Claudia, O. (2017). Does capital structure influence company profitability?. Studies in Business and Economics, 12(3), 50-62. https://doi.org/10.1515/sbe-2017-0036

Modigliani, F., \& Miller, M. H. (1958). The cost of capital, corporate finance and the theory of investment. The American Economic Review, 48, 261-297.

Modigliani, F., \& Miller, M. H. (1963). Corporate income taxes and the cost of capital: A correction. The American Economic Review, 53(3), 433-443.

Mugoša, A. (2015). The determinants of capital structure choice: Evidence from Western Europe. BEH - Business and Economic Horizons, 11(2), 76-95. http://dx.doi.org/10.15208/beh.2015.07

Myers, S. C. (1984). The capital structure puzzle. The Journal of Finance, 39(3), 575-592. https://doi.org/10.1111/j.1540-6261.1984.tb03646.x

Myers, S. C. (2001). Capital structure. The Journal of Economic Perspectives, 15(2), 81-102. 
Myers, S. C., \& Majluf, N. S. (1984). Corporate financing and investment decisions when firms have information that investors do not have. Journal of Financial Economics, 13(2), 187-221. https://doi.org/10.1016/0304-405X(84)90023-0

Pais, M. A., \& Gama, P. M. (2015). Working capital management and SMEs profitability: Portuguese evidence. International Journal of Managerial Finance, 11(3), 341-358. https://doi.org/10.1108/JJMF-11-2014-0170

Pederzoli, C., Thoma, G., \& Torricelli, C. (2013). Modelling credit risk for innovative SMEs: The role of innovation measures. Journal of Financial Services Research, 44(1), 111-129. https://doi.org/10.1007/s10693-012-0152-0

Pepur, S., Ćurak, M., \& Poposki, K. (2016). Corporate capital structure: The case of large Croatian companies. Economic Research-Ekonomska Istraživanja, 29(1), 498-514. https://doi.org/10.1080/1331677X.2016.1175726

Pindado, J., Rodrigues, L., \& de la Torre, C. (2006). How does financial distress affect small firms' financial structure?. Small Business Economics, 26, 377-391. https://doi.org/10.1007/s11187-005-4845-8

Pinková, P. (2012). Determinants of capital structure: Evidence from the Czech automotive industry. ACTA Universitatis Agriculturae et Silviculturae Mendelianae Brunensis, 60(7), 217-224.

Psillaki, M., \& Daskalakis, N. (2009). Are the determinants of capital structure country or firm specific?. Small Business Economics, 33(3), 319-333. https://doi.org/10.1007/s11187-008-9103-4

Ramalho, J. J. S., \& da Silva, J. V. (2009). A two-part fractional regression model for the financial leverage decisions of micro, small, medium and large firms. Quantitative Finance, 9(5), 621-636. https://doi.org/10.1080/14697680802448777

Rauh, J. D., \& Sufi, A. (2010). Capital structure and debt structure. The Review of Financial Studies, 23(12), 4242-4280. https://doi.org/10.1093/rfs/hhq095

Šarlija, N., \& Harc, M. (2012). The impact of liquidity on the capital structure: A case study of Croatian firms. Business Systems Research, 3(1), 30-36. https://doi.org/10.2478/v10305-012-0005-1

Schäfer, D., Werwatz, A., \& Zimmermann, V. (2004). The determinants of debt and (private) equity financing: The case of young, innovative SMEs from Germany. Industry and Innovation, 11(3), 225-248. https://doi.org/10.1080/1366271042000265393

Serghiescu, L., \& Văidean, V.-L. (2014). Determinant factors of the capital structure of a firm- an empirical analysis. Procedia Economics and Finance, 15, 1447-1457. https://doi.org/10.1016/S2212-5671(14)00610-8

Sogorb-Mira, F. (2005). How SME uniqueness affects capital structure: Evidence from a 1994-1998 Spanish data panel. Small Business Economics, 25(5), 447-457. https://doi.org/10.1007/s11187-004-6486-8

Strýčková, L. (2015). Factors determining the corporate capital structure in the Czech Republic from the respective of business entities. $E \quad a \quad M$ : Ekonomie a Management, 18(2), 40-56. http://dx.doi.org/10.15240/tul/001/2015-2-004

Van Caneghem, T., \& Van Campenhout, G. (2012). Quantity and quality of information and SME financial structure. Small Business Economics, 39(2), 341-358. https://doi.org/10.1007/s11187-010-9306-3

Vatavu, S. (2015). Determinants of return on assets in Romania: A principal component analysis. Timisoara Journal of Economics and Business, 8(1s), 32-47. https://doi.org/10.1515/tjeb-2015-0003

Warner, J. B. (1977). Bankruptcy costs: Some evidence. The Journal of Finance, 32(2), 337-347.

Westhead, P., \& Storey D. J. (1997). Financial constraints on the growth of high technology small firms in the United Kingdom. Applied Financial Economics, 7(2), 197-201. https://doi.org/10.1080/096031097333763 